\title{
Optimized Approach to Improve Classification of Wrist Movements via Electromyography Signals
}

\author{
Almon Chai \\ Faculty of Engineering, Computing and \\ Science, \\ Swinburne University of Technology \\ Sarawak Campus, \\ Kuching, Sarawak, Malaysia \\ achai@swinburne.edu.my
}

\author{
Evon Wan Ting Lim \\ Faculty of Engineering, Computing and \\ Science, \\ Swinburne University of Technology \\ Sarawak Campus, \\ Kuching, Sarawak, Malaysia
}

\author{
Phei Chin Lim \\ Faculty of Computer Science and \\ Information Technology, \\ Universiti Malysia Sarawak \\ Kota Samarahan, Sarawak, Malaysia \\ pclim@unimas.my
}

\begin{abstract}
An optimized approach aiming to improve classification accuracy of wrist movements via electromyography (EMG) signals is presented here. EMG signals of the different types of wrist movements are obtained from the NINAPRO database. Useful features are extracted from the EMG signals via the waveform length method. The developed optimized classification system contains two main modules, known here as (i) optimized neural network module and (ii) movement prediction module. The optimized neural network module is made up of multiple 2-class neural networks. During Stage 1 Classification, a group of neural network (named NNG S1) is formed after analyzing the sensitivity computed from the training outcomes of each neural network. A new group of neural network (named NNG_S2) is later formed in Stage 2 Classification after initial elimination via Stage 1 Classification. Further analysis is performed via the movement prediction module to predict the final outcome of the classification. The overall average classification accuracy achieved via the optimized classification system is $8.3 \%$ higher than the conventional neural network. The results validate that the optimized classification system performs better than the conventional neural network, providing more accurate signals for manipulating of exoskeleton for rehabilitation purposes.
\end{abstract}

Keywords-electromyography, wrist movement, neural network, classification

\section{INTRODUCTION}

Electromyography (EMG) signals can be gathered from muscles when the muscles are contracted. Activities related to the muscles can be analyzed from the EMG signals. These signals are preferred by researchers in the biomedical and rehabilitation field, especially to identify human movements [1-6]. Classification of human movements via EMG signals allows these signals to be used as controller inputs to manipulate robots or exoskeletons that mimic the human movements [7-11]. Different signals can correspond to different inputs in order to replicate the different movements made during an arm or hand movement.

Exoskeletons, when used for rehabilitation, help patients during arm or hand recovery. Patient hand movement, when weak, do not provide the control system sufficient signal input. This is where classified EMG signals can be applied as control signals. However, noises such as ambient noise, inherent noise, motion artefact and inherent instability [12] are present in EMG signals and therefore proper filtering and processing of signals are required. Raw EMG signals are weak, where the amplitude is typically in the range of 0 to 10 $\mathrm{mV}$ [13]. The frequency lies between $10 \mathrm{~Hz}$ to $500 \mathrm{~Hz}$ while the dominant energy falls between $50 \mathrm{~Hz}$ to $150 \mathrm{~Hz}$ [14]. Noises can be eliminated by applying filters to the EMG signals.
Normalization of the EMG signals is also one of the important steps when comparison of signals between different individuals is required [15-16]. Useful features need to be extracted from the EMG signals before the signals can be analyzed [17]. Numerous feature extraction methods had been introduced and preferred by researchers such as the mean absolute value (MAV), root mean square (RMS), simple square integral (SSI), zero crossing (ZC), waveform length (WL), variance (VAR) and fast Fourier Transform (FFT) [1824]. Several classifiers are preferred by researchers for the classification of human movements via EMG signals. There are numerous examples of classifiers ranging from artificial neural network (ANN), radial basis function neural network (RBFNN), deep neural network (DNN), support vector machine (SVM), logistic model tree (LMT), simple logistic regression (SLR) to linear discriminant analysis (LDA) [2530].

An optimized classification system that aims to improve classification accuracy is developed and validated in this paper. A total of 40 cases of experiments regarding the classification of 6 types of wrist movements are conducted. Each case consists of testing dataset from subject that is excluded from the training dataset to study the ability of the system to classify EMG data from different individuals. The classification outcomes obtained via the optimized classification system is compared to the classification outcomes obtained via a conventional neural network to validate the performance of the optimized classification system.

\section{Methodology}

\section{A. Data Preparation Flow}

EMG data acquired from 12 electrodes regarding the wrist movements from 40 subjects are used to evaluate the performance of the proposed optimized classification system. The EMG data are downloaded from the NINAPRO Database. According to [31], 8 channels of EMG signals are gathered from 8 electrodes equally located around the forearm and 4 channels of EMG signals are gathered from 4 specific muscle locations. The 6 basic wrist movements include wrist supination, wrist pronation, wrist flexion, wrist extension, wrist ulnar deviation and wrist radial deviation. The illustration of the movements can be found in Fig. 1 .

Since comparison of EMG signals between different subjects is required, the filtered raw EMG signals are normalized. The signals are then rectified via full-wave rectification to preserve all components of the signals. Then, the waveform length (WL) method is used to extract useful features from the processed signals for further analysis. The 